\title{
INSULATED MODULAR AUXILIARY POWER SOURCE CONCEPT WITH MULTIPLE OUTPUTS
}

\author{
Gustavo Lambert, Fabiana Seidel, Luan Vinícius Fiorio, Yales R. De Novaes \\ Santa Catarina State University, Joinville-Santa Catarina, Brazil \\ Department of Electrical Engineering \\ Electric Power Processing Group - nPEE \\ e-mail: gustavolambert@ outlook.com, fabianaseidel@ outlook.com
}

\begin{abstract}
This paper presents an insulated auxiliary power source with multiple outputs based on a two-stage structure, where the first-stage operates as a controlled current source and the second-stage as an active rectifier. The second-stage is replicated for each load, where the output voltage is independently controlled by a hysteresis band controller integrated to the auxiliary power source's circuit. Insulation between the multiple stages is achieved by a medium-frequency transformer for each output. The main aim of this auxiliary power supply is to feed modular multilevel converters in laboratory applications that run at the lower range of medium-voltage dc. Theoretical analysis of the auxiliary power supply is presented and verified experimentally for the converter's two main modes of operation.
\end{abstract}

Keywords - Auxiliary power source, Modular multilevel converter, Multiple-output.

\section{INTRODUCTION}

The development of the Modular Multilevel Converter (MMC) concept brought an entirely new range of possibilities for medium and high voltage applications in the last decade(s) [1]-[4]. By the means of this concept, semiconductor's voltage and current level technology limits have been overcome without the series connection of semiconductors [5]. To achieve such behavior, these converters are built by the association of submodules (SMs) to reduce the voltage or current stress among them.

Nonetheless, this concept exchanges semiconductor's technology limitations into converter complexity which is verified by the converter control [6], [7], operation [2], [8], data communication [9]-[11] and extra functionalities in the SM. Further than these issues and besides the SM's main power circuit, the MMC's SM have other secondary circuits, just as crucial, which are responsible for the signal conditioning, the gate-drives signaling, the SM protection and other. These secondary elements typically use energy from an Auxiliary Power Source (APS), which is the main concern of this paper.

The MMC requirements for an APS can be divided into two categories. The first is intrinsic to the APS, such as its power capability, the input and output voltage levels, noise rejection and efficiency. The second is dependent on the MMC application and operation which may impact: the required

Manuscript received 28/09/2018; first revision 15/11/2018; accepted for publication 12/02/2019, by recommendation of Editor Marcello Mezaroba. http://dx.doi.org/10.18618/REP.2019.2.0043 insulation level among different references, the number of APS cells, the operation modes (supplying energy in any condition for a critic black-start for example), the amount of data exchanged, and other even more specific requirements. Thus, the APS concept development is driven by two main concerns. The first is the voltage-level insulation between the auxiliary source and each floating SM. And, the second is to provide energy even when the converter has zero voltage on its bus. Due to the requirement of communication at all times and control of other secondary functions, the need for power in the SM is critical. Because of it, solutions based on the SM bus to supply the auxiliary circuits, such as the ones proposed in [12] and [13], are out of choice. Then, it is concluded that an external source is required.

The isolation concern can be straightforward addressed by the use of a transformer. However, to supply multiple loads, a transformer with multiple windings overlapped may face difficulties to achieve high levels of insulation with low volume and cost due to the windings proximity. Also, the parasitic elements create a low impedance path for highfrequency currents that is severe for the converter operation. In addition, multiple-winding multiple-output solutions have to face the cross-regulation characteristics [14] or use an extra regulator for each output [15], [16] accordingly to the load requirements. These constraints leads to one transformer per SM solution although it may increase the SM volume and cost.

The proposed one transformer per output isolation approach is already verified in the literature [17]. Although similar, this alternative uses a LLC resonant circuit topology and focus to address the existing high-voltage insulation difficulties. The resonant converter in such application benefit from the high gain, the reduced switching losses and the neat use of parasitic elements. However, to avoid difficulties as the reactive energy circulation, the gain variation with aging and parametric variations, and the design and control effort of such converter, this paper investigates a different approach.

More specifically, this paper proposes an insulated auxiliary source partially modular with multiple outputs that aims to meet the requirements of different multilevel modular converters prototypes developed in our laboratory. The insulation is provided by a single transformer per SM and rated for the lower range of MVDC. The only two windings transformers are expected to ease the insulation complexity when compared to multiple-winding transformers. Although one transformer per SM is needed, the SM volume is not expected to be much increased due to the low power. The proposed APS also features an independent output control per SM to avoid cross-regulation. In this paper, the main operation 
modes are identified, explained, discussed and compared before the later verification by experiments.

\section{AUXILIARY POWER SOURCE CONCEPT}

The proposed APS is shown in Figure 1 supplying a singlephase MMC. In the first-stage there is a sinusoidal current source (external power source) which is responsible to provide energy to all cells. The active second-stages are connected to the current source through their primary winding connected in series. Although the APS is shown with only one current source, separated loops with different current sources could be used to increase the APS modularity (for example one for each MMC's arm) and/or reliability (if one fails, the other remains operating). In total, at least one first-stage and $2 \mathrm{~N}$ second-stage converters are necessary for a single-phase MMC. Where $\mathrm{N}$ is the number of SMs in a MMC's arm and two MMC's arm form an MMC's leg.

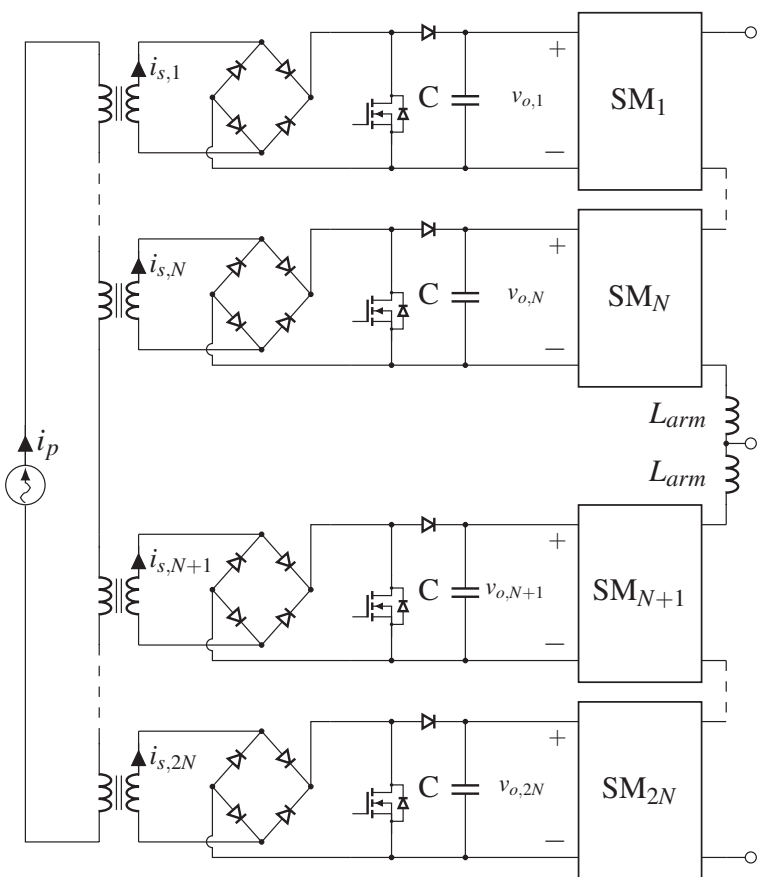

Fig. 1. Circuit diagram of the auxiliary power source connected to a MMC leg.

From the concept, the second-stage (converter cell) is chosen to be independent of any external control. This is achieved by having its own regulator circuit that enables the converter cell to operate from a complete black start condition.

In the proposed arrangement (series connection), the most likely condition where one converter cell could disable the other is if, for any reason, the primary current loop is open. Assuming that the transformer's input coil may fail open, to improve the reliability, a bypass switch could be added in parallel with this coil. Since the primary winding current is controlled, all power cells are immune to other power cell short-circuit.

Among the main features of the proposed auxiliary source, it is highlighted the simplicity of the converter cell, the inherited tolerance to short-circuits and parasitic inductances over the current loop, its reliability, modularity and consequent expandability, and the reduced cost.

\section{CONVERTER CELL}

\section{A. Elements, Operation and Control}

The converter cell is composed by the following elements: medium frequency transformer, full-bridge diode rectifier, semiconductor switch $(Q)$, diode $\left(D_{o}\right)$ and capacitor $\left(C_{o}\right)$. The circuit diagram showing these elements connections is presented in Figure 2, where the source's current $i_{p}$ reflected to the secondary is $i_{s}(t)=i_{s, p k} \cdot \sin (\omega t)$.

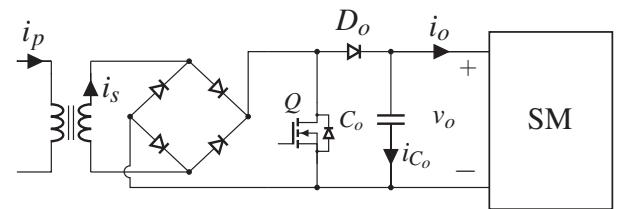

Fig. 2. Circuit diagram of the converter cell.

The converter operation is summarized by the two possible states of $Q$. When $Q$ is "off", the rectified current is supplied to the capacitor and load through $D_{o}$. If $Q$ is "on", the rectified current is conducted through Q and the load is supplied by the energy stored in the capacitor $C_{o}$. To reach steady-state operation, the main requirement for the average value of the rectified current $\left(I_{S}\right)$ is to be higher than the average value of the load current $\left(I_{o}\right)$. By assuming the rectifier to be ideal, the average value of the rectified current is $I_{s}=2 \cdot i_{s, p k} / \pi$.

One may notice that the converter power cell resembles the boost converter, only the boost inductor is missing. However, even if one considers the leakage inductance as the boost inductor, this converter operation is different due to the current source nature. For instance, there is no charge and discharge process controlled by the switch $\mathrm{Q}$ as it is verified in the boost converter. Here, the parasitic inductor current is determined by the current source. Nevertheless, the transformer's leakage inductance is considered sufficiently small to be neglected as well as the phenomena related to it.

Fulfilled the operation requirements, it is observed that the output voltage may behave differently accordingly to the values of: $K=i_{s, p k} / I_{o}, C_{o}$ and the equivalent resistive load $\left(R_{o}\right)$. As it is presented in the next sections, two main modes are observed: 1) High-switching frequency and 2) Lowswitching frequency. The mentioned switching frequency is referred to $Q$. Also, the adopted control strategy may influence some characteristics.

This paper consider the output voltage $\left(v_{o}\right)$ being controlled by a hysteresis method. In this method, the comparison of $v_{o}$ with the maximum output voltage $\left(v_{o, \max }\right)$ and the minimum output voltage $\left(v_{o, \min }\right)$ defines the $Q$ command signal. Thus, the switch Q is commanded to close if $v_{o}$ is greater than $v_{o, \max }$ and it is commanded to open if $v_{o}$ becomes lower than $v_{o, \min }$. Other than stated, the switch keeps its current state. Using this technique, both switching frequency and duty cycle varies according to $i_{s}, C_{o}$ and $R_{o}$.

This APS must be supplied by its own output voltage since there is no other energy source. Because of its design, in the lack of energy, the switch Q remains open which allows the output to be supplied by the current source as soon as current starts flowing into the converter cell. One of many possibilities for a circuit to behave as stated is shown in Figure 3. 


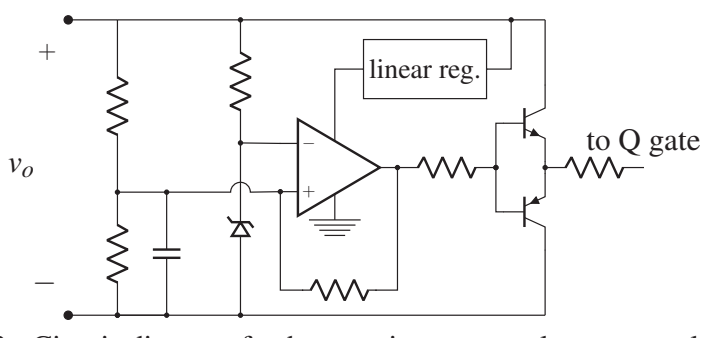

Fig. 3. Circuit diagram for hysteresis output voltage control and to drive $Q$.

\section{B. High-Switching Frequency Mode}

By definition, in the High-Switching Frequency Mode (HSFM) $Q$ turns ON and OFF more than once in a half-cycle of the sinusoidal current source $i_{s}$. This phenomenon is easily identified in Figure 4. The main advantage of this mode is the reduced output filter since the predominant ripple in the output voltage is at high-frequency.

a)

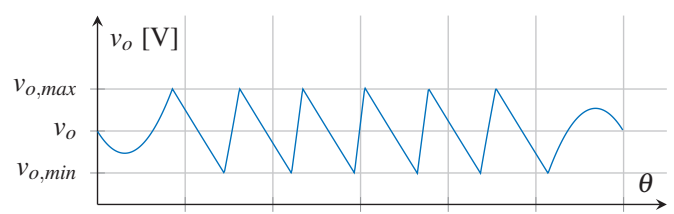

b)

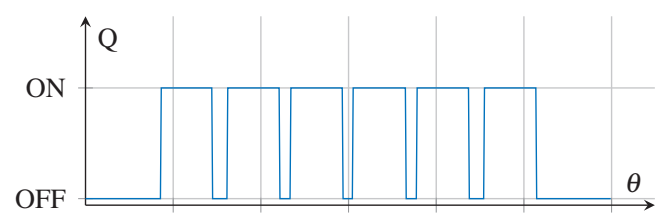

c)

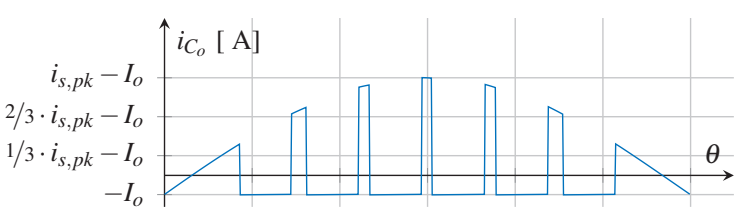

d)

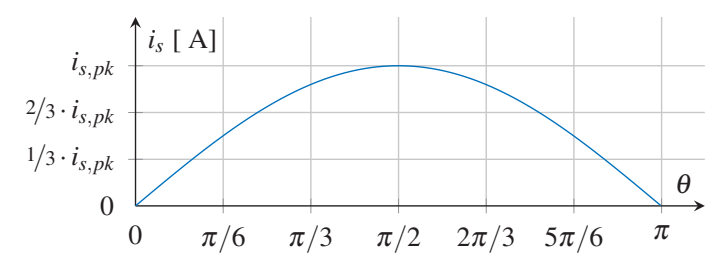

Fig. 4. HSFM simulated waveforms: a) output voltage $\left(v_{o}\right)$, b) main switch gate signal and c) input current $\left(i_{s}\right)$.

The charging $\left(T_{1}\right)$ and discharging $\left(T_{2}\right)$ periods associated to the voltage ripple are obtained assuming that the capacitor's voltage derivative can be approximated by the difference between the final value and the initial value $\left(\Delta v_{o}\right)$. Thus,

$$
\begin{gathered}
T_{1}(\omega t)=\frac{C_{o} \Delta v_{o}}{i_{s, p k} \cdot \sin (\omega t)-I_{o}} . \\
T_{2}=\frac{C_{o} \Delta v_{o}}{I_{o}} .
\end{gathered}
$$

This information allows to obtain the switching frequency

$$
f_{s}(\omega t)=\frac{1}{T_{1}+T_{2}}=\frac{i_{s}(\omega t) I_{o}-I_{o}^{2}}{i_{S}(\omega t) C_{o} \Delta v_{o}}=\frac{I_{o}\left[1-I_{o} /\left(i_{s, p k} \cdot \sin (\omega t)\right)\right]}{C_{o} \Delta v_{o}} .
$$

Which can be rewritten by assuming the ratio $K=I_{o} / i_{s, p k}$ and the periods $T_{1}(\omega t)$ and $T_{2}$ as

$$
f_{s}(\omega t)=\frac{I_{o}}{C_{o} \Delta v_{o}} \cdot\left(1-\frac{K}{\sin (\omega t)}\right) .
$$

Equation (4) clearly shows the switching frequency dependency on $K, C_{o}$, and $\Delta v_{o}$. Using (3), the switching frequency relation with the load variation is presented in Figure 5. Another characteristic noted from Figure 5 is the zero frequency interval, which means $Q$ "OFF". This happens because $i_{S}$ is lower than $i_{o}$ during the angle interval $\theta_{u v}$ (uncontrolled voltage angle interval). As a result, it causes the output voltage to be uncontrolled until $i_{s}$ rises beyond $i_{o}$. Using the rectified current as angle reference, the uncontrolled interval is defined to start at $\pi-\arcsin (K)$ and to end at $\arcsin (K)$. Consequently, the total interval can be obtained by $2 \cdot \arcsin (K)$.

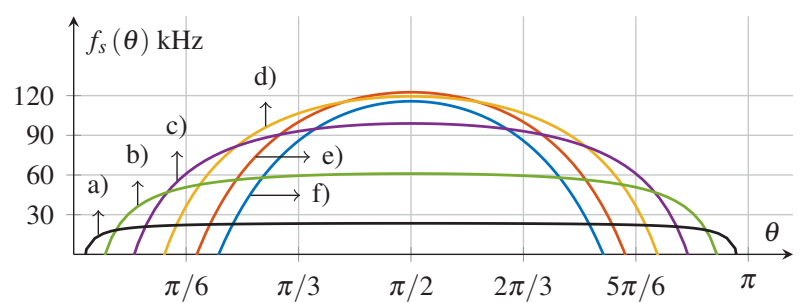

Fig. 5. Switching frequency variation along $\theta$ for different $\mathrm{K}$ values due to load variation. Curves: a) $\theta_{u v}=2.86^{\circ}$ and $K=0.05$, b) $\theta_{u v}=$ $8.31^{\circ}$ and $K=0.1445$, c) $\theta_{u v}=16.09^{\circ}$ and $K=0.2772$, d) $\theta_{u v}=$ $24.19^{\circ}$ and $K=0.4098$, e) $\theta_{u v}=32.85^{\circ}$ and $K=0.5424$, and f) $\theta_{u v}=$ $38.68^{\circ}$ and $K=0.6250$.

The importance of considering the uncontrolled period remains in the fact that the output voltage may fall beyond the specified lower voltage limit. Thus, the output capacitor must have enough energy stored to withstand the mentioned period or the $\mathrm{K}$ value should be lower to reduce this period. This is exemplified by the two cases shown in Figure 6.

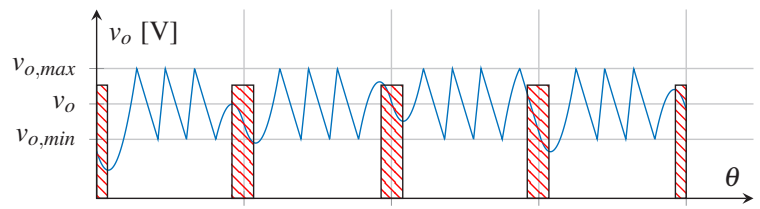

a)

b)

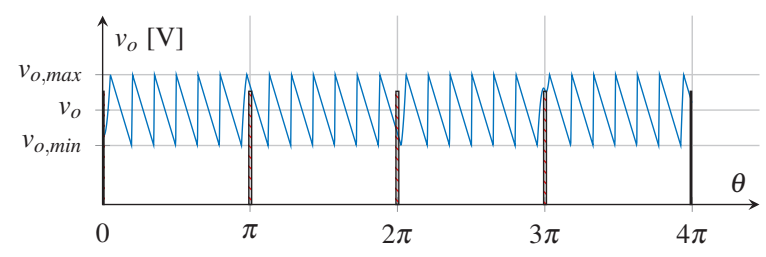

Fig. 6. Output voltage for: a) $K=0.2$ and b) $K=0.025$. The regions where the input current becomes lower than the output current are hatched.

The voltage drop $\left(\Delta v_{c, u v}\right)$ along the uncontrolled voltage region can be obtained by the capacitor initial voltage $\left(v_{c, i}\right)$ and the integral of the capacitor current during this period as

$$
\Delta v_{c, u v}+v_{c, i}=v_{c, f}=v_{c, i}+\frac{1}{\omega C} \int_{-\theta_{u v}}^{\theta_{u v}} i_{s}-i_{o} \mathrm{~d} \theta .
$$


This integral has the problem of the output current being a function of the voltage still unknown. A solution by approximation is to suppose $i_{o}=\left(v_{c, i}-\Delta v_{c, u v} / 2\right) / R_{o}$. Thus,

$$
\begin{gathered}
\int_{-\theta_{u v}}^{\theta_{u v}} i_{s, p k} \cdot|\sin (\theta)| \mathrm{d} \theta=2 \cdot i_{s, p k} \cdot\left[1-\cos \left(\theta_{u v}\right)\right] . \\
\int_{\theta_{u v}}^{\theta_{u v}} \frac{\left(v_{c, i}-\Delta v_{c, u v} / 2\right)}{R_{o}} \mathrm{~d} \theta=\frac{\left(v_{c, i}-\Delta v_{c, u v} / 2\right)}{R_{o}} \cdot 2 \cdot \theta_{u v} .
\end{gathered}
$$

Finally, applying the obtained terms and simplifying the final equation, the voltage drop will be

$$
\Delta v_{c, u v}=\frac{2 R_{o} i_{s, p k} \cdot\left[1-\cos \left(\theta_{u v}\right)\right]}{R_{o} \omega C_{o}-\theta_{u v}}-\frac{2 v_{c, i} \cdot \theta_{u v}}{R_{o} \omega C_{o}-\theta_{u v}} .
$$

where the main difficult associated with this equation is to find $v_{c, i}$. Although the exact value is difficult to obtain, its range is known to be between $v_{o, \min }$ and $v_{o, \max }$. This approximation is acceptable because the difference between $v_{o, \max }$ and $v_{o, \min }$ is small, which implies in a small error.

In its turn, the duty cycle is defined as $D=T_{2} \cdot f_{s}$, which occurs when $Q$ is "ON". The expression of $D(\omega t)$ is derived applying the values of (2) and (4),

$$
\begin{gathered}
D(\omega t)=\frac{C_{o} \Delta v_{o} f_{s}}{I_{o}}=\frac{C_{o} \Delta v_{o}}{I_{o}} \cdot\left(\frac{i_{s, p k} \sin (\omega t) I_{o}-I_{o}^{2}}{i_{s, p k} \sin (\omega t) C_{o} \Delta v_{o}}\right) . \\
D(\omega t)=\frac{i_{s, p k} \sin (\omega t)-I_{o}}{i_{s, p k} \sin (\omega t)}=\frac{\sin (\omega t)-K}{\sin (\omega t)} .
\end{gathered}
$$

which is only valid for $0 \leq D(\omega t) \leq 1$ and $K \leq 2 / \pi$. The plot of (10) is shown in Figure 7 for several K values.

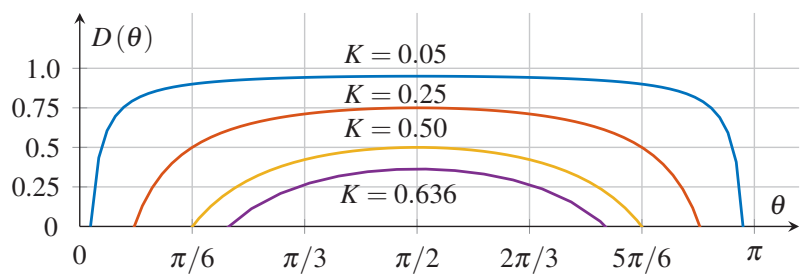

Fig. 7. Q duty cycle along $\theta$ for different ratios of K.

\section{Low-Switching Frequency Mode}

The Low-Switching Frequency Mode (LSFM) occurs when the load is supplied continuously by the rectified current $i_{s}$ for a period longer than the half-cycle, during this interval $Q$ is "off" and $D_{o}$ is "on". This behavior is exemplified by the simulated waveforms in Figure 8.

Assuming that the $i_{S}$ can be simplified by its average value, the sum of charging and discharging periods $\left(T_{1}+T_{2}\right)$ results in

$$
\begin{gathered}
T_{1}+T_{2}=T_{1}+T_{2}=\frac{I_{s} \cdot C_{o} \cdot \Delta V_{o}}{I_{o} \cdot\left(I_{s}-I_{o}\right)} \\
T_{1}+T_{2}=\frac{i_{s, p k} \cdot 2 / \pi \cdot C_{o} \cdot \Delta V_{o}}{I_{o} \cdot\left(i_{s, p k} \cdot 2 / \pi-I_{o}\right)}=\frac{C_{o} \cdot \Delta V_{o}}{I_{o} \cdot(1-\pi / 2 \cdot K)} .
\end{gathered}
$$

For the condition where $T_{1}+T_{2}$ should be larger than one a)
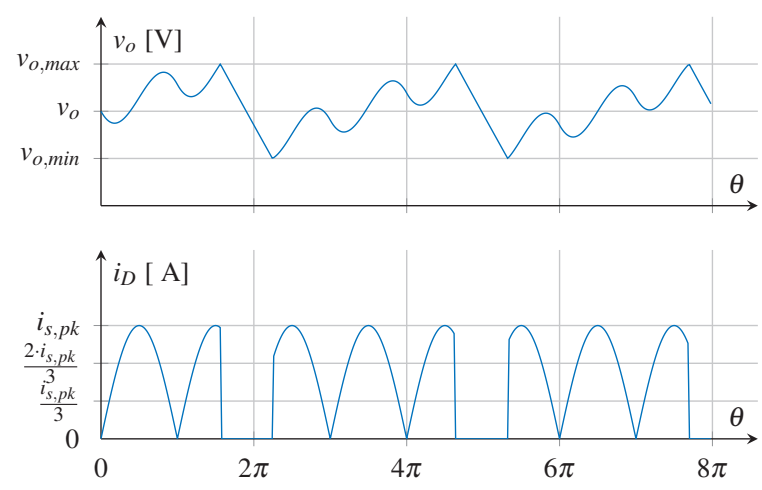

Fig. 8. LSFM waveforms: a) Output voltage and b) output diode currents.

half-cycle of the input current

$$
\frac{\pi}{\omega}=\frac{1}{2 \cdot f}<T_{1}+T_{2}=\frac{C_{o} \cdot \Delta V_{o}}{I_{o} \cdot(1-\pi / 2 \cdot K)} .
$$

Since all variables are always positive for the analysis, the output capacitance that guarantees the LSFM should be selected as

$$
C_{o}>\frac{\pi}{\omega} \cdot \frac{I_{o} \cdot\left(I_{s}-I_{o}\right)}{I_{s} \cdot \Delta V_{o}}=\frac{\pi}{\omega} \cdot \frac{I_{o} \cdot(1-K \cdot \pi / 2)}{\Delta V_{o}} .
$$

where $K \leq 2 / \pi$ still applies.

\section{Almost Constant Switching Frequency Condition}

The almost constant switching frequency operation occurs when the rectified current has an average value much larger than the average value of the load's current. In this case, the output ripple has a saw-tooth waveform. Then, it is possible to assume that the switching frequency is constant because the discharge period is approximately equal to $T_{s}$. In Figure 9, it is shown the output voltage and the diode current $\left(i_{D_{o}}\right)$ waveforms for this special case.

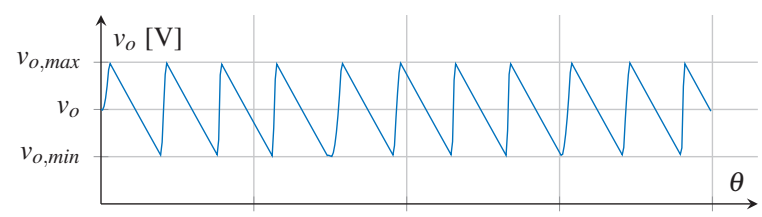

a)

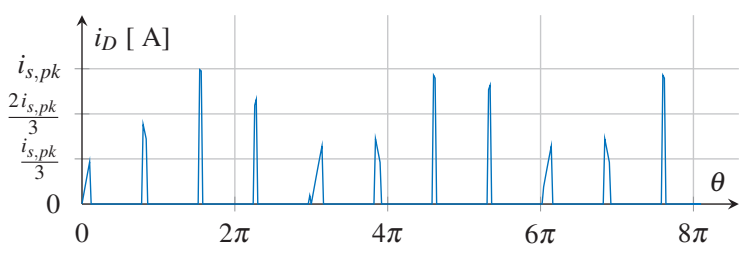

Fig. 9. Almost constant switching frequency waveforms: a) Output voltage and b) output diode currents .

This special case may occur for both HSFM and LSFM because the only condition necessary is $T_{1}$ being much smaller than $T_{2}$. Thus, $T_{2}$ can be bigger or smaller than $\pi / \omega$.

\section{E. Power Cell Apparent Power}

The apparent power $\left(S=v_{s, r m s} \cdot i_{s, r m s}\right)$ in the transformer is obtained for the highest power condition. This happens when none of the rectified current is bypassed by $Q$ and the average 
output voltage still remains in the specified limits. Then, the apparent power is

$$
S=V_{o} \cdot \sqrt{\frac{1}{\pi} \int_{0}^{\pi}\left[i_{s, p k} \cdot \sin (\theta)\right]^{2} \mathrm{~d} \theta}=\frac{V_{o} \cdot i_{s, p k}}{\sqrt{2}} .
$$

Where the ratio between the output active power and the apparent power, neglecting the output voltage ripple, is

$$
\frac{p_{o, a v g}}{S}=\frac{V_{o} \cdot i_{s, p k} \cdot 2}{\pi} \cdot \frac{\sqrt{2}}{V_{o} \cdot i_{s, p k}}=\frac{2 \sqrt{2}}{\pi} .
$$

\section{F. Current Source Considerations}

For the APS due to the power level required and cost, we chose to use a Half-Bridge converter (HB) which is fed by a full-bridge diode rectifier as shown in Figure 10. Both converters are well known since decades. This inverter is operated as a sinusoidal current source by the means of controlling the filter inductor $\left(L_{f}\right)$ current. In addition, a small filter capacitor $\left(C_{f}\right)$ is used to control the propagated high frequency noise that might cause interference in the submodule.

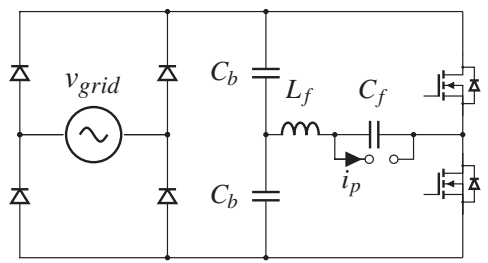

Fig. 10. Circuit diagram of half-bridge converter operated as the current source.

The frequency of the sinusoidal current $\left(i_{p}\right)$ is a degree of freedom for the designer. By changing its value, the size and volume of each transformer and filters along the converter are affected. Nonetheless, this frequency is supposed to be in the medium frequency range (here defined as hundreds of Hertz up to kilo Hertz).

\section{G. HSFM and LSFM Comparison}

Although defining the best mode of operation is dependent on external needs, it is securely fair to state the advantages and disadvantages of each operation mode. In case of the HSFM, the higher frequency ripple of the output voltage is advantageous for filtering. Consequently, the smaller capacitance results in reducing the volume, the weight and possibly the cost of the converter. However, it becomes a disadvantage because the voltage drop is more pronounced during the $\theta_{u v}$. At last, the higher switching frequency is expected to reduce the cell efficiency and can potentially require the use of extra actions to guarantee the junction temperature within the semiconductors limits, such as a bigger heat sink.

For the LSFM, the main difference when compared to the HFSM is the bigger capacitance. Basically, this is disadvantageous in opposition to the advantage reasons given for the HFSM. But, since there is more energy stored in the capacitor, the voltage drop effect during $\theta_{u v}$ in the output voltage becomes negligible. Further than these, there might be a problem with the low frequency ripple associated with this mode, which may be difficult to filter. This side effect, however, can be dealt by choosing a narrower hysteresis band to reduce even more the ripple amplitude. Last, since the switching losses are significantly smaller than in the previous modes, it is expected the efficiency to be higher.

\section{EXPERIMENTAL RESULTS AND DISCUSSION}

The proposed APS is verified experimentally for different configurations to present the HSFM and LSFM. All experiments are conducted using the parameters presented in Table I.

TABLE I

Auxiliary Power Source Design Parameters

\begin{tabular}{c|c|c|c}
\hline \hline Parameter & Value & Parameter & Value \\
\hline$v_{o}$ & $15 \mathrm{~V}$ & $\Delta v_{o}$ & $400 \mathrm{mV}$ \\
\hline$N_{p} / N_{s}$ & $1 / 10$ & $i_{s, p k}$ & $0.3 \mathrm{~A}$ \\
\hline \hline
\end{tabular}

For experimental validation with a linear load, the APS cell is rated for a maximum output power of $2.5 \mathrm{~W}$. The constructed cell is shown in Figure 11. This setup diagram is the composition of the circuits shown in Figure 2 and Figure 3. Where the main components are the Schottky diodes SS16 (60 V,1 A), the MOSFET IRF7313, the $5 \mathrm{~V}$ linear regulator (LM78L05) and the comparator LMV7219M5. For the transformer, the considered insulation requirement is $1 \mathrm{kV}$. To address it, a bakelite bobbin and primary and secondary windings displaced $3.5 \mathrm{~mm}$ apart were sufficient measures. If higher voltage isolation was necessary, the solution would basically consist in substituting the insulating materials in the critical parts. One of those is the wiring, which could have better insulating material, such as the PTFE, or could have the windings as they are but coated with some resin. Another possibility is to use another material for the bobbin spool. Apart from substitutions of dielectric material, other core and spool that allows increasing the distance between the windings is another solution, but it implies in a bigger footprint.
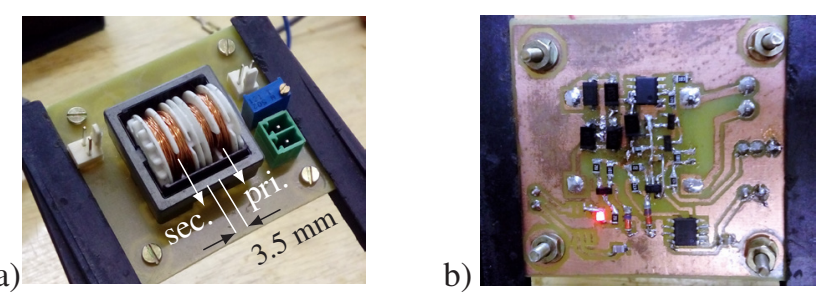

Fig. 11. APS power cell prototype: a) top view and b) bottom view. Input to output capacitance is $5.58 \mathrm{pF}$ measured at $1 \mathrm{kHz}$. The board dimensions are $50 \mathrm{~mm} \times 61 \mathrm{~mm}$ and the displacement between primary and secondary windings is $3.5 \mathrm{~mm}$.

The first configuration under experiment uses $C_{o}=2.33 \mu \mathrm{F}$ and $R_{o}=750 \Omega$. For the chosen input current, the resulting set point is an HSFM with ratio $K \approx 0.0689$. The main waveforms are shown in Figure 12. The theoretical switching period around the peak value of $i_{S}$ is $\approx 50 \mu$ s, which was obtained using (4). In the experiment, this period is $\approx 40 \mu \mathrm{s}$. The explanation on the difference remains partially on the analog components used for the hysteresis band control which causes the comparison to occurs in different voltage level than 
specified. In special, the capacitance variation of the ceramic capacitors due to voltage bias. Another cause of the difference is the parasitic elements not considered during the theoretical analysis such as the Equivalent Series Inductance (ESL) of the capacitors and the parasitic elements from the layout. Since the calculated periods are dependent on the passive elements, any variation on these and the obtained results are critically affected.

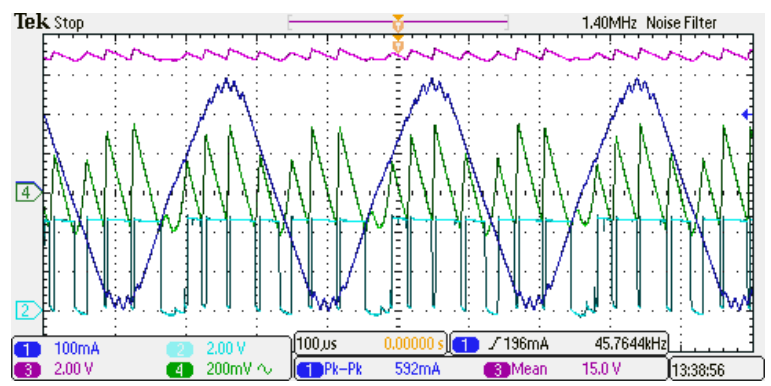

Fig. 12. HSFM waveforms for $C_{o}=2.33 \mu \mathrm{F}$ and $R_{o}=750 \Omega$ : 1) input current $\left(i_{S}\right)$ in blue, 2) gate signal in light blue, 3$)$ output voltage $\left(v_{o}\right)$ in pink and 4$)$ output voltage $\left(v_{o}\right)$ using ac coupling in green.

Still on HSFM, the $K$ is increased to $\approx 0.1969$ to stress the aforementioned not controlled voltage region. This is shown in Figure 13 where the theoretical period of $\theta_{u v} \approx 11^{\circ}$ is verified. But the theoretical voltage drop of $200 \mathrm{mV}$, in the experiment is measured $400 \mathrm{mV}$.

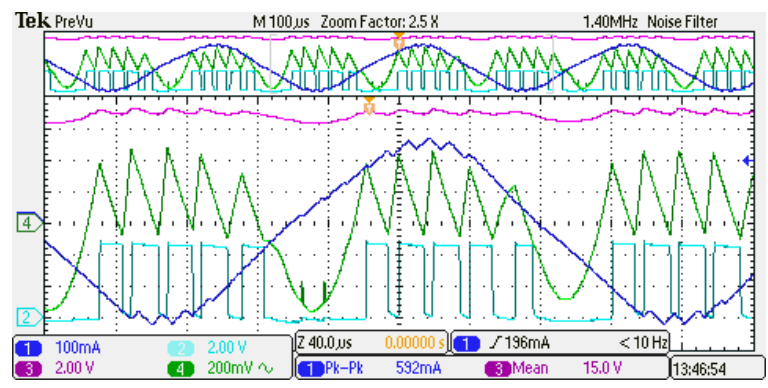

Fig. 13. HSFM waveforms for $C_{o}=2.33 \mu \mathrm{F}$ and $R_{o}=262.6 \Omega$ : 1) input current $\left(i_{s}\right)$ in blue, 2) gate signal in light blue, 3) output voltage $\left(v_{o}\right)$ in pink and 4$)$ output voltage $\left(v_{o}\right)$ using ac coupling in green.

The black start of the APS for the HSFM is shown in Figure 14. It takes in total approximately $8 \mathrm{~ms}$ to reach steadystate. However, most of this time was due to the current source start-up process.

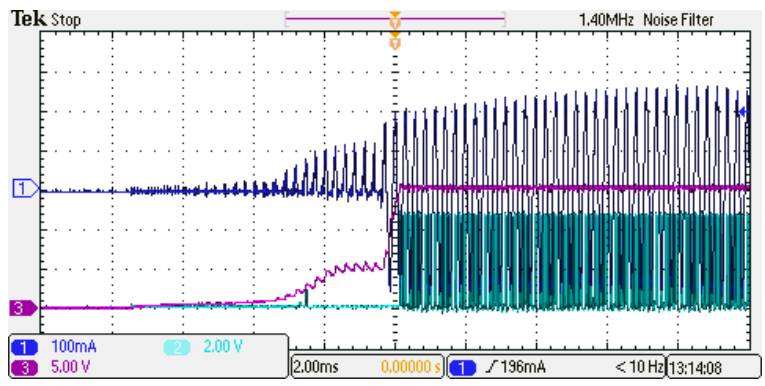

Fig. 14. HSFM waveforms during the black star for $C_{o}=2.33 \mu \mathrm{F}$ and $\left.R_{O}=262.6 \Omega: 1\right)$ input current $\left(i_{S}\right)$ in blue, 2) gate signal in light blue, 3$)$ output voltage $\left(v_{o}\right)$ in pink and 4$)$ output voltage $\left(v_{o}\right)$ using ac coupling in green.
The LSFM is verified experimentally in a configuration where $C_{o}=152.33 \mu \mathrm{F}, R_{o}=96 \Omega$ and $K \approx 0.539$. As presented in theory, the output voltage has an inherited ripple due to the sinusoidal rectified current. Also, due to the LSFM, a second ripple according to the hysteresis band. The LSFM is specially interesting because even in a condition, where the $\theta_{u v}$ is large, the output voltage does not drop significantly due to the larger capacitor when compared to the HSFM.

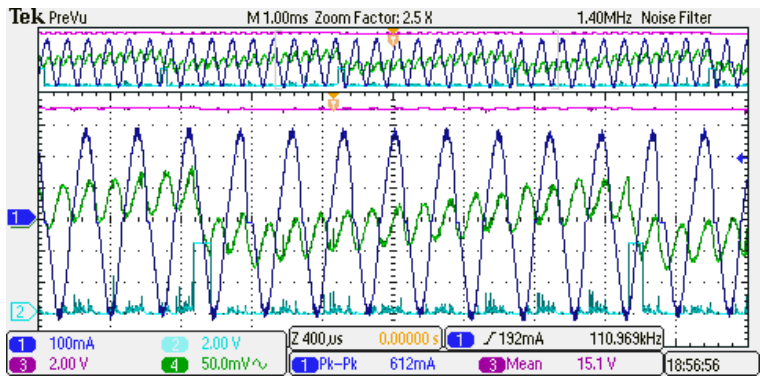

Fig. 15. LSFM waveforms for $C_{o}=152.33 \mu \mathrm{F}$ and $R_{o}=96 \Omega$ : 1) input current $\left(i_{S}\right)$ in blue, 2) gate signal in light blue and 3) output voltage $\left(v_{o}\right)$ in pink.

Last, the black start for the LSFM configuration is presented in Figure 16. Due to the larger output capacitor, the charging procedure lasts for about $40 \mathrm{~ms}$. Even for the same load, the output capacitor is critical and always makes the LSFM slower for load steps when compared to the HSFM.

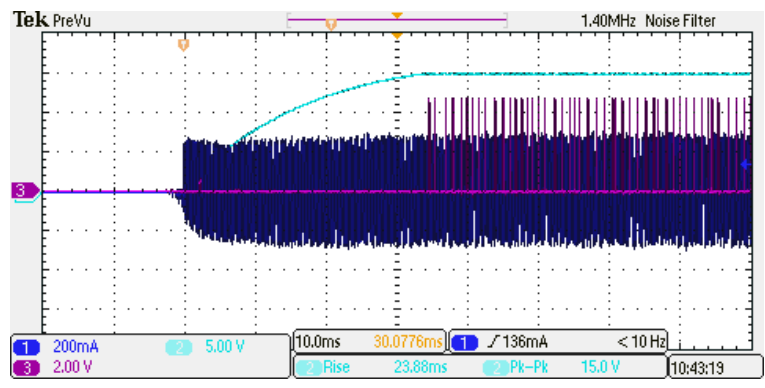

Fig. 16. LSFM waveforms during the black star for $C_{o}=154 \mu \mathrm{F}$ and $\left.R_{o}=108 \Omega: 1\right)$ input current $\left(i_{S}\right)$ in blue, 2$)$ output voltage $\left(v_{o}\right)$ in light blue and 3) gate signal in pink.

\section{CONCLUSIONS}

This paper presented a modular APS intended to feed multiple loads independently. This APS was designed to feed several submodules of a modular multilevel converter in the laboratory. Among the main features, it is highlighted the isolation capability which can be easily extended. Also, the reduced amount of elements and the individual regulation of each output.

The APS operation is described through the paper in terms of the two main modes of operation: the HSFM and the LSFM. Both modes of operation were verified through experimentation. As it has been shown by simulation and by experiments, these two modes have different dynamic characteristics which should be considered to match with the load requirements.

Although the current level of development allows the APS to operate with a breakdown isolation voltage up to $4 \mathrm{kV}$, value obtained through HIPOT (High Potential) test, 
further development is necessary before the implementation in practical MVDC converters. In special, a careful selection criteria should be adopted when choosing the transformer insulation materials and the protection elements.

\section{ACKNOWLEDGMENTS}

The authors would like to thank CAPES, FAPESC and FITEJ for the funding and the support to our research laboratory (nPEE).

\section{REFERENCES}

[1] A. Lesnicar, R. Marquardt, "A new modular voltage source inverter topology", in European Power Electronics Conference (EPE), Toulouse, France, September 2-4 2003.

[2] S. Debnath, J. Qin, B. Bahrani, M. Saeedifard, P. Barbosa, "Operation, Control, and Applications of the Modular Multilevel Converter: A Review", IEEE Transactions on Power Electronics, vol. 30, no. 1, pp. 37-53, Jan 2015, doi:10.1109/TPEL.2014.2309937.

[3] V. K, J. L. Rao, G. Bopparaju, Y.-J. Hafner, D. Shearer, P. Lundberg, "Design Aspects of MTDC Grids with Integration of Renewable Energy Sources", in CIGRE, Technical Exhibition, Aug. 2018.

[4] X. Guo, M. Deng, K. Wang, "Characteristics and performance of Xiamen VSC-HVDC transmission demonstration project", in IEEE International Conference on High Voltage Engineering and Application (ICHVE), pp. 1-4, Sept 2016, doi: 10.1109/ICHVE.2016.7800677.

[5] A study $n$ monitoring technology for multiterminal $\pm 500 \mathrm{kV}$ VSC-HVDC power transmission system, March 2017, doi:10.1109/IAEAC.2017.8054361.

[6] A. E. Leon, S. J. Amodeo, "Modeling, control, and reduced-order representation of modular multilevel converters", Electric Power Systems Research, vol. 163, pp. 196 - 210, May, 2018, doi:https://doi.org/10. 1016/j.epsr.2018.05.024.

[7] D. Karwatzki, A. Mertens, "Generalized Control Approach for a Class of Modular Multilevel Converter Topologies", IEEE Transactions on Power Electronics, vol. 33, no. 4, pp. 2888-2900, April 2018, doi:10.1109/ TPEL.2017.2703917.

[8] G. J. M. de Sousa, A. d. S. Dias, J. A. Alves, M. L. Heldwein, "Modeling and control of a Modular Multilevel Converter for medium voltage drives rectifier applications", in IEEE 24th International Symposium on Industrial Electronics (ISIE), pp. 10801087, June 2015, doi:10.1109/ISIE.2015.7281622.

[9] L. Mathe, P. D. Burlacu, R. Teodorescu, "Control of a Modular Multilevel Converter With Reduced Internal Data Exchange", IEEE Transactions on Industrial Informatics, vol. 13, no. 1, pp. 248-257, Feb 2017, doi: 10.1109/TII.2016.2598494.

[10] J. Lago, G. J. M. Sousa, M. L. Heldwein, "Digital control/modulation platform for a modular multilevel converter system", in Brazilian Power Electronics Conference, pp. 271-277, Oct 2013, doi:10.1109/ COBEP.2013.6785127.
[11] H. Tu, S. Lukic, "Comparative study of PES Net and SyCCo bus: Communication protocols for modular multilevel converter", in IEEE Energy Conversion Congress and Exposition (ECCE), pp. 1487-1492, Oct 2017, doi:10.1109/ECCE.2017.8095966.

[12] T. Modeer, S. Norrga, H.-P. Nee, "High-Voltage Tapped-Inductor Buck Converter Utilizing an Autonomous High-Side Switch", IEEE Transactions on Industrial Electronics, vol. 62, no. 5, pp. 28682878, May 2015, doi:10.1109/TIE.2014.2365157.

[13] P. J. Grbovic, "Master/Slave Control of InputSeries- and Output-Parallel-Connected Converters: Concept for Low-Cost High-Voltage Auxiliary Power Supplies", IEEE Transactions on Power Electronics, vol. 24 , no. 2 , pp. 316 - 328, February 2009.

[14] C. Ji, M. Smith, K. M. Smedley, K. King, "Cross regulation in flyback converters: analytic model and solution", IEEE Transactions on Power Electronics, vol. 16, no. 2, pp. 231-239, March 2001, doi:10.1109/ 63.911147.

[15] J. Kim, J. Lee, G. Moon, “Zero-Voltage Switching Multioutput Flyback Converter With Integrated Auxiliary Buck Converter", IEEE Transactions on Power Electronics, vol. 29, no. 6, pp. 3001-3010, June 2014, doi:10.1109/TPEL.2013.2276443.

[16] M. Tahan, D. Bamgboje, T. Hu, "Flyback-Based Multiple Output dc-dc Converter with Independent Voltage Regulation", in 9th IEEE International Symposium on Power Electronics for Distributed Generation Systems (PEDG), pp. 1-8, June 2018, doi: 10.1109/PEDG.2018.8447884.

[17] D. Peftitsis, M. Antivachis, J. Biela, "Auxiliary Power Supply for Medium-Voltage Modular Multilevel Converters", 17th European Conference on Power Electronics and Applications, pp. 1-11, September 2015.

\section{BIOGRAPHIES}

Gustavo Lambert (SOBRAEP and IEEE student member) was born in Palmas, Paraná in 21/01/1989. He received the B.S. and M.S. degrees in Electrical Engineering from the Santa Catarina State University (UDESC), Joinville, Brazil, in 2012 and 2015, respectively. He is currently pursuing the Dr. degree at Santa Catarina State University. His research interests include the design and control of modular multilevel converter topologies for HVDC and MVDC applications.

Fabiana Seidel was born in Rio das Antas, Santa Catarina Brazil in 24/06/1996. She received the bachelor degree in electrical engineering from the Santa Catarina State University (UDESC) in 2018 and currently is pursuing her master degree student at the same institution. She works as a trainee researcher on the Researcher and Technologic Innovation area at WEG Industries. Her areas of interest are power electronics, electronic control systems, and systems monitoring sensors.

Luan Vinícius Fiorio born in 09/07/1997 in Concórdia, Santa Catarina, Brazil, is a bachelor degree student in the Santa 
Catarina State University (UDESC). His areas of interest are audio electronics, power electronics, system identification, and artificial intelligence.Between 2017 and 2018 he was a power electronics researcher at UDESC and he's currently studying the digital emulation of vacuum-tube preamplifiers using artificial intelligence and its use with a class D power amplifier.

Yales R. De Novaes (SOBRAEP and IEEE member) was born in Indaial, Santa Catarina, Brazil, in 1974. In 1999 he received the B.S. degree in electrical engineering from FURB - Regional University of Blumenau, Santa Catarina, Brazil. The M. Eng. and Dr. degrees were obtained from the Power
Electronics Institute (INEP) of the Federal University of Santa Catarina (UFSC) in 2000 and 2006, respectively. During 2001, he worked as a researcher engineer at the same institute. From 2006 to 2008 was a post-doctoral fellow at the Industrial Electronics Laboratory (LEI) at École Polytechnique Fédéral de Lausanne (EPFL), Lausanne, Switzerland. From 2008 to 2010 worked as scientist with the Power Electronics Systems group at ABB Corporate Research Center, Daetwill, Switzerland. Currently he is an associate professor at Santa Catarina State University (UDESC), Joinville, Brazil, and coordinator of CMEAR (Research Cell on Microgrids of Alternative and Renewable Energies). 